\title{
Plasma enhanced metalorganic chemical vapor deposition of amorphous aluminum nitride
}

\author{
H. Harris, ${ }^{\text {a) }}$ N. Biswas, H. Temkin, and S. Gangopadhyay \\ Physics Department, Texas Tech University, Lubbock, Texas 79409 \\ M. Strathman \\ Thin Film Analysis, Sunnyvale, California 94086
}

(Received 8 June 2001, accepted for publication 28 August 2001)

\begin{abstract}
Plasma enhanced deposition of amorphous aluminum nitride (AlN) using trimethylaluminum, hydrogen, and nitrogen was performed in a capacitively coupled plasma system. Temperature was varied from 350 to $550{ }^{\circ} \mathrm{C}$, and pressure dependence of the film structure was investigated. Films were characterized by Fourier transform infrared, Rutherford backscattering (RBS), ellipsometry, and x-ray diffraction (XRD). The films are amorphous in nature, as indicated by XRD. Variations in the refractive index were observed in ellipsometric measurements, which is explained by the incorporation of carbon in the films, and confirmed by RBS. Capacitance-voltage, conductancevoltage $(G-V)$, and current-voltage measurements were performed to reveal bulk and interface electrical properties. The electrical properties showed marked dependence on processing conditions of the AlN films. Clear peaks as observed in the $G-V$ characteristics indicated that the losses are predominantly due to interface states. The interface state density ranged between $10^{10}$ and $10^{11} \mathrm{eV}^{-1} \mathrm{~cm}^{-2}$. Annealing in hydrogen resulted in lowering of interface state density values.

(c) 2001 American Institute of Physics. [DOI: 10.1063/1.1413484]
\end{abstract}

\section{INTRODUCTION}

The interest in aluminum nitride (AlN) has increased tremendously in recent years. Epitaxial AlN has been deposited on silicon as an intermediate layer for the growth of GaN on bulk silicon. ${ }^{1}$ Sputtered AlN has been studied extensively, including growth mechanics and characterization, ${ }^{2-4}$ while remote plasma molecular beam epitaxy methods have been proven to provide high quality AlN. ${ }^{5}$ The large band gap of $6.2 \mathrm{eV}$ makes AlN interesting for optoelectronics (for high crystal quality AlN), as well as antireflective coatings.

With the scaling of device sizes, introduction of tunneling currents in the insulator becomes the primary concern for any device application. Alternatively, the required capacitance can be achieved by using an insulator with a high dielectric constant without having to reduce the thickness of the insulator film. Due to its high dielectric constant $(\sim 8-10)$, AlN films can replace $\mathrm{SiO}_{2}$ (dielectric constant $\sim 3.9$ ), the predominate dielectric used in the past. However, a dielectric to be considered as a replacement to $\mathrm{SiO}_{2}$ should also possess superior surface passivation properties with the substrate semiconductor. An estimate of interface trap density is thus crucial. Interface traps originate from defects such as dangling bonds at the insulator-silicon interface with energy states in the siliconforbidden band gap and are dependent on the chemical composition of the interface. ${ }^{6}$ Among the several methods listed in theory, conductance technique, as proposed by Nicollian and Goetzberger, ${ }^{7,8}$ is by far the most effective method for carrying out the interfacial characterization.

a)Electronic mail: rusty.harris@ttu.edu
Plasma enhanced metalorganic chemical vapor deposition (PE-MOCVD) of AlN using a conventional parallel plate has been the focus of very few studies, ${ }^{9}$ while the studies of interface properties have been cursory at best. ${ }^{10}$ This article presents the properties of PE-MOCVD AlN. In depth electrical properties are presented and the interface properties of AlN with Si are explored.

\section{EXPERIMENT}

Films were deposited on $\mathrm{Si}(100)$ on the grounded electrode of a parallel plate plasma enhanced chemical vapor deposition system, with $5 \mathrm{~cm}$ spacing and a fixed frequency of $13.56 \mathrm{MHz}$. Trimethylaluminum, nitrogen, and hydrogen were supplied at leak rates of 3,6 , and $3 \mathrm{sccm}$, respectively, for all samples. Pressure was varied from 100 to $600 \mathrm{mT}$, and the temperature was set at either 350 or $550^{\circ} \mathrm{C}$. Prior to deposition, wafers were cleaned by the modified Shiraki method $^{11}$ to produce a single atomic hydrogen-passivated surface.

Fourier transform infrared (FTIR) spectra were taken using a Perkin-Elmer model 1600 spectrometer with $8 \mathrm{~cm}^{-1}$ resolution. A Shimatsu model 2400 PC UV-visible spectrometer was used to obtain optical absorption data. Rutherford backscattering was performed to determine the atomic concentration of the films. Thickness was measured using a profilometer and a Rudolph Instruments AutoEL ellipsometer was used to find the refractive index. $2 \Theta$ scans were collected in a Philips X'pert X-ray diffraction system. No significant diffraction peak for AlN was observed at $\sim 36^{\circ}$, indicating that the films are amorphous in nature.

Capacitance-voltage $(C-V), \quad$ conductance-voltage $(G-V)$, and current-voltage $(I-V)$ measurements were 
TABLE I. Process parameters and measured data for $a$-AlN samples of this study.

\begin{tabular}{cccccccr}
\hline \hline Sample & $\begin{array}{c}\text { Pressure } \\
(\mathrm{mT})\end{array}$ & $\begin{array}{c}\text { Temperature } \\
\left({ }^{\circ} \mathrm{C}\right)\end{array}$ & $\begin{array}{c}\text { Power } \\
(\mathrm{W})\end{array}$ & $\begin{array}{c}\text { Al/N } \\
\text { ratio }\end{array}$ & $\% \mathrm{C}$ & $\begin{array}{c}\text { Thickness } \\
(\mathrm{nm})\end{array}$ & Index $n$ \\
\hline $\mathrm{A}$ & 200 & 350 & 60 & 0.9 & 0 & 170 & 2.05 \\
$\mathrm{~B}$ & 600 & 550 & 15 & 1.12 & 20 & 240 & 1.86 \\
$\mathrm{C}$ & 600 & 550 & 30 & 0.89 & 37.2 & 280 & 1.75 \\
$\mathrm{D}$ & 600 & $550(900)$ & 30 & 1.09 & 40.7 & 280 & \\
\hline
\end{tabular}

used to evaluate AlN films deposited on silicon and on silicon coated with TiN/Al/Ti. The metal-insulatorsemiconductor (MIS) structure was comprised of metal electrodes evaporated on AlN/Si. $C-V$ and $G-V$ analysis was performed on metal/dielectric/silicon structures using an HP 4275A LCR meter. Selective $C-V$ curves were analyzed to find the interface density of states, $D_{\text {it }}$.

\section{A. Film characterization}

We prepared 18 samples by varying total process pressure, rf power, and substrate temperature. Table I lists the three samples that are the focus of this study. Included in the table are the process parameters, $\mathrm{Al} / \mathrm{N}$ ratio, at. \% $\mathrm{C}$, and index of refraction.

The sample with the highest rf power and lowest process pressure is sample A. Consequently, this sample showed the lowest amount of carbon, below the detection limit of $\pm 3 \%$ for RBS. This is attributed to a higher degree of ionization in the plasma, and subsequent desorption of $\mathrm{CH}$ and $\mathrm{CH}_{2}$ species from the film. This sample is nitrogen rich, having an $\mathrm{Al} / \mathrm{N}$ ratio slightly below 1 , and its index was measured at 2.047, which correlates well with AlN from other sources. ${ }^{12,13}$ The optical band gap of the sample is $5.6 \mathrm{eV}$, close to the crystalline AlN band gap value of $6.2 \mathrm{eV} .{ }^{12}$ The decrease is attributed to some of the $\mathrm{Al}$ and $\mathrm{N}$ bonded in the amorphous structure.

An increase in pressure, combined with a decrease in the power, and an increase in the substrate temperature, as seen from sample B to sample C, produced a likewise increase in the percent of carbon incorporated in the film. It is assumed that the increase in pressure increases the concentration of carbon species in the plasma and in the film. The end result is a decrease in the index of refraction, from just over 2 to 1.9 for sample B and to 1.75 for sample C.

Fourier transform infrared spectra of the films deposited on both (100) and (111) silicon was acquired, and the corresponding silicon baseline was subtracted. After the baseline interference was removed, the spectra displayed a peak at about $667 \mathrm{~cm}^{-1}$. The spectra for samples A-D are shown in Fig. 1. The peak located at $1110 \mathrm{~cm}^{-1}$ is associated with the oxygen interstitial in silicon, and shows up due to a slight mismatch in the baseline subtraction. The small series of peaks at $2340 \mathrm{~cm}^{-1}$ are from carbon dioxide within the IR instrument.

The dominant peak at $667 \mathrm{~cm}^{-1}$ for all the samples is assigned to the AlN transverse optical mode (TO). ${ }^{14-17}$ The full width at half maximum (FWHM) for this peak for samples $\mathrm{A}, \mathrm{B}$, and $\mathrm{C}$ are 114,220 , and $245 \mathrm{~cm}^{-1}$, respectively. Incorporation of carbon in samples $\mathrm{B}$ and $\mathrm{C}$ may pro- duce a high degree of disorder in these samples, which may be responsible for a significant increase in the FWHM.

For sample B another peak emerges at $1795 \mathrm{~cm}^{-1}$ that may be associated with olefinic or aromatic carbon. This vibration mode is not IR active in amorphous carbon films. However, the presence of an $\mathrm{Al}$ or nitrogen neighbor may break the symmetry and make this mode IR active.

Sample C surprisingly produced more C content, almost double that of sample B. In this sample, the AlN peak at 667 $\mathrm{cm}^{-1}$ is evident, but two new peaks emerge: the peak at 2200 $\mathrm{cm}^{-1}$ and the broadband between 1100 and $1700 \mathrm{~cm}^{-1}$. The peak at $2120 \mathrm{~cm}^{-1}$ is associated with $\mathrm{Al}-\mathrm{H}$ stretch, ${ }^{14}$ while the broadband is the combination of $s p^{3}$ and $s p^{2}$ carbon, as well as $\mathrm{OH}$ and NH modes. ${ }^{14,16}$ The broad peak in the 30003750 range is attributed to the combination of $\mathrm{NH}$ and $\mathrm{OH}$, as suggested by Lorentz et al. ${ }^{15}$ Since there was no $\mathrm{O}$ in the precursors and an ultrahigh vacuum was achieved before deposition $\left(5 \times 10^{-7}\right.$ Torr $)$, the oxygen was absorbed postdeposition. Higher rf power produces more $\mathrm{H}$ in the amorphous matrix that, upon exposure to atmosphere, is easily hydrolyzed.

Sample D is the spectra of sample C annealed at $900{ }^{\circ} \mathrm{C}$ in vacuum. RBS indicates that the $\mathrm{H}$ content decreased from $14 \%$ to $2.5 \%$. The IR peaks at $2200 \mathrm{~cm}^{-1}(\mathrm{Al}-\mathrm{H})$ and 3500 $\mathrm{cm}^{-1}(\mathrm{OH}, \mathrm{NH})$ were not present in the IR spectra of the annealed sample. The thickness of the sample did not change appreciably after annealing, but the relative magnitude of the $667 \mathrm{~cm}^{-1}$ band increased considerably. This is attributed to an increased density of $\mathrm{Al}-\mathrm{N}$ bonds produced as an after

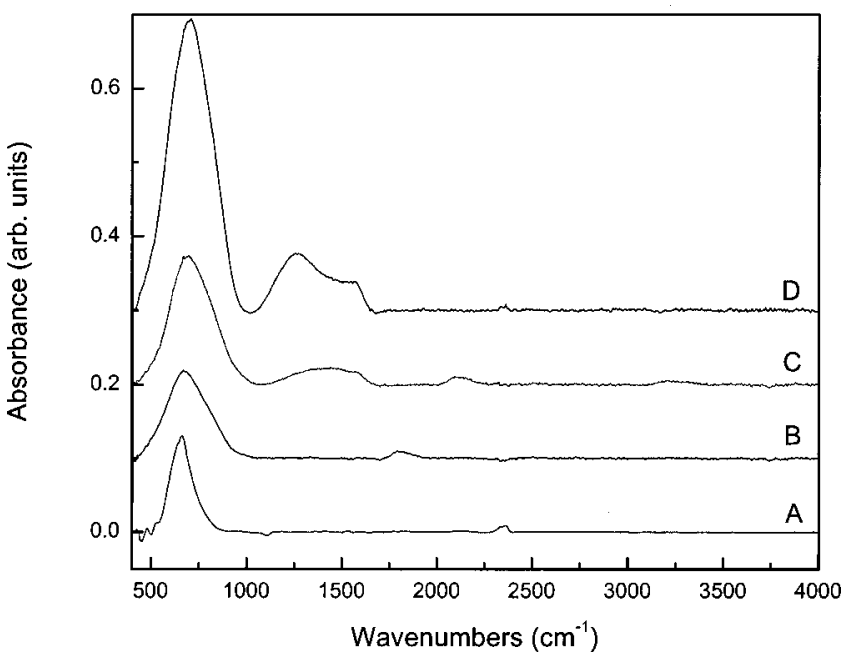

FIG. 1. FTIR spectra of samples A-D. 


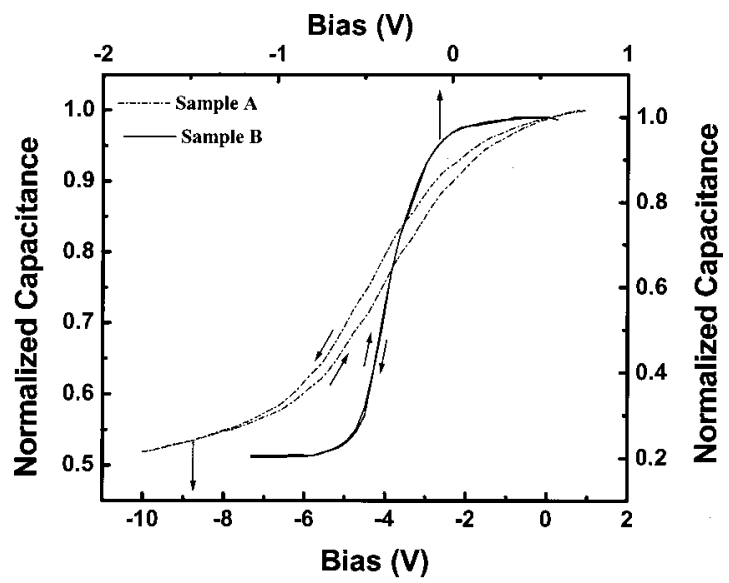

FIG. 2. Hysteresis effect in sample A (dash-dot) and in sample B (solid) at $1 \mathrm{MHz}$.

effect of $\mathrm{H}$ desorption during annealing. Furthermore, the broadband from 1100 to $1700 \mathrm{~cm}^{-1}$ shows more structure and increased intensity from the removal of the $\mathrm{OH}$ and $\mathrm{NH}$, as evident in the missing $3500 \mathrm{~cm}^{-1}$ mode.

\section{B. Electrical characterization}

Electrical characterization was performed on all samples. Two samples of particular interested are presented: samples A and B. Sample A represents the best quality AlN film in terms of purity, as indicated in the previous section, and the electrical properties of this sample are a generalized representation of other samples. Sample B has an intermediate amount of atomic carbon, $20 \%$.

The conductance technique involves point-by-point determination of interface state density throughout the depletion region. A conductance peak is observed because of the ac loss due to capture and emission of carriers by the interface states. From the admittance, the equivalent parallel conductance $\left(G_{p}\right)$ is extracted. To calculate the interface state density ( $\left.D_{\text {it }}\right)$ using $G_{p}$, two models were used: (1) the single time constant model and (2) the continuum of states model. ${ }^{7}$ According to the single time constant model, $G_{p} / \omega$ is written as

$$
\frac{G_{p}}{\omega}=\frac{q D_{\mathrm{it}} \omega \tau}{\left(1+\omega^{2} \tau^{2}\right)},
$$

where $D_{\mathrm{it}}$ is the density of interface states, $q$ is the electronic charge, $\tau$ is the time constant of the interface state, and $\omega$ is the frequency. When a continuum of interface states is present the above expression changes to

$$
\frac{G_{p}}{\omega}=\frac{q D_{\text {it }}}{2 \omega \tau} \ln \left(1+\omega^{2} \tau^{2}\right) .
$$

The maximum value of $G_{p} / \omega$ occurs at $\omega \tau=1$ for Eq. (1) and at $\omega \tau=1.98$ for Eq. (2). The time constants of the interface traps can thus be determined using the aforesaid condition on $\omega \tau$.

Figure 2 shows the $C-V$ characteristics of samples A and $\mathrm{B}$, along with the hysteresis effect for measurements done at $1 \mathrm{MHz}$. As can be seen, the $C-V$ curves are shifted

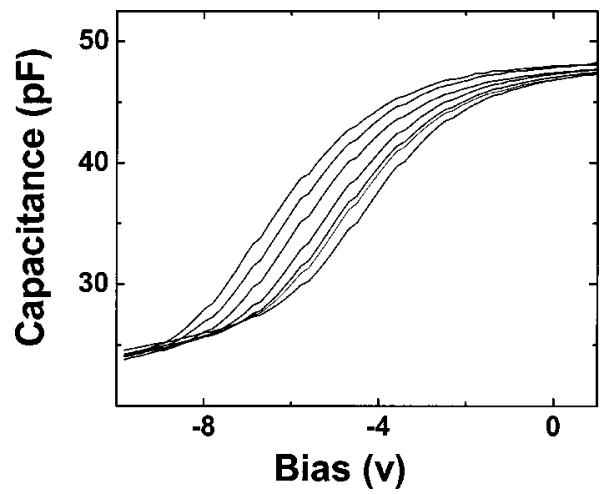

FIG. 3. Frequency dependence of the $C-V$ curves for sample A. Frequencies shown are $10,20,40,100,200$, and $400 \mathrm{kHz}$ and $1 \mathrm{MHz}$ left to right.

towards negative biases. The shift of the $C-V$ curve towards negative biases indicates the presence of positively charged ions at the insulator-silicon interface. The magnitude of hysteresis for sample A $(\sim 0.36 \mathrm{~V})$ is approximately ten times more than that for sample B $(\sim 30 \mathrm{mV})$. The nature of hysteresis is opposite for both the samples. The counterclockwise loop of the hysteresis for sample A can be attributed to charge polarization in the insulator. Charge polarization is well known in other dielectrics on $\mathrm{Si}^{18-21}$ The clockwise nature of hysteresis as observed for sample B is typical of majority carrier injection from semiconductor to insulator with subsequent trapping. Such trapping could occur in the insulator bulk near the interface if unsaturated bonds and/or vacancies are present. The deep-depletion nature of the curves, after threshold is achieved, in sample A indicates that minority charge carriers are lost either by recombination or injection whereas the constant capacitance reached for sample B indicates that inversion has been achieved. ${ }^{7}$

Figure 3 represents the capacitance-voltage $(C-V)$ curves measured at the indicated frequencies for sample A. $C-V$ curves for sample A are stretched about the voltage axis and are marked with minimal frequency dispersion in the accumulation region $(<2 \%)$, about $41 \%$ dispersion in the depletion region and no dispersion in the deep-depletion region. Frequency dispersion of capacitance in accumulation is also observed in many other MIS structures. ${ }^{22-24}$ In most cases, however, the dispersion is abnormally large. In our case the small dispersion in the accumulation region is predominantly due to the frequency dispersion of the dielectric constant of the insulator, which was confirmed by $C-V$ measurements on the relevant Al/AlN/TiN (MIM) structure at different frequencies. MIM structures are not subjected to any back contact and so are representative of true insulator properties. Frequency dispersion of capacitance in accumulation is also observed by several other groups for AlN samples. ${ }^{25,26} \mathrm{~A}$ flatband voltage of $-4.86 \mathrm{~V}$ was recorded at a flatband capacitance of $42.52 \mathrm{pF}$ using the $C-V$ curve at $10 \mathrm{kHz}$.

The stretch about the voltage axis is due to the response of the interface traps to the applied dc bias. The dispersion in depletion is due to the response of interface traps to applied frequency. If the time constants $\tau$ of the interface traps are comparable to the frequency $f$ of the small signal $(2 \tau \pi f$ 

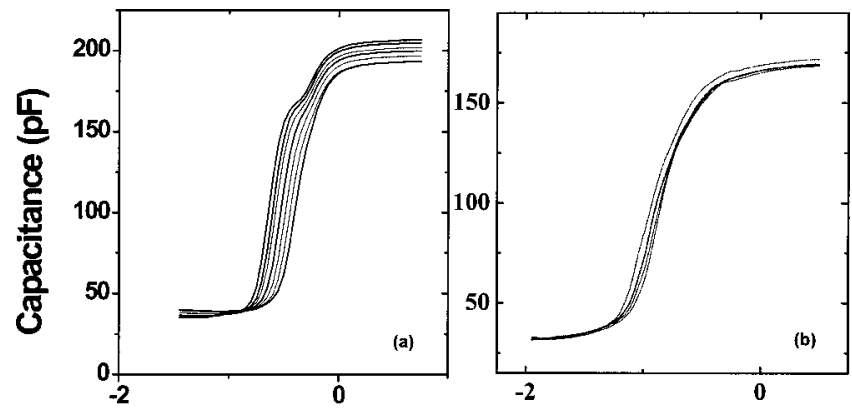

Bias ( $)$

FIG. 4. Frequency dependence of the $C-V$ curves for (a) sample B and (b) sample B annealed in hydrogen at frequencies of 10, 20, 40, 100, 200, and $400 \mathrm{kHz}$ and $1 \mathrm{MHz}$ left to right.

1) the interface traps make a contribution to the total capacitance such that the threshold capacitance increases with decreasing frequency. ${ }^{27}$

The scope of this investigation was extended to include studies involving annealing the samples in hydrogen gas. For thermally grown $\mathrm{SiO}_{2}$ on $\mathrm{Si}$, annealing in hydrogen is known to improve the interface, and a considerable reduction in the $D_{\text {it }}$ values is observed. ${ }^{7,28}$ As is discussed next, similar results were obtained by annealing AlN films in hydrogen. All the samples were annealed in hydrogen gas at $250^{\circ} \mathrm{C}$ for $1 \mathrm{~h}$ and 500 mTorr gas pressure. Marked improvement in the interfacial properties was observed for all the samples resulting in a much lower value of $D_{\text {it }}$ at the midgap. Few samples were chosen, however, for detailed studies.

Figures 4(a) and (b) represent the $C-V$ curves for sample B and sample B annealed in hydrogen at the indicated frequencies. The $C-V$ curves in Fig. 4(a) are characterized by a sharp transition from accumulation to inversion and small flatband voltage shift. Frequency dispersion in accumulation was calculated to be $\sim 6 \%$. The small frequency dispersion of capacitance in the accumulation region is once again due to the dispersion of the insulator dielectric constant. The presence of a "ledge" can be noted in the lower frequency curves of Fig. 4(a). When reducing the applied bias from large positive biases in accumulation towards the depletion region, the capacitance is characterized by a sharp drop followed by a deep-depletion-like characteristic at a bias voltage of $-0.3 \mathrm{~V}$ (for measurements done at $10 \mathrm{kHz}$ ). The deep-depletion-like characteristics were confirmed by plotting $1 / C^{2}$ versus biases. Such a plot yielded a straight line in the bias range of -0.35 to $-0.45 \mathrm{~V}$. Above an applied bias of $-0.45 \mathrm{~V}$, the capacitance follows the depletion and inversion sequence. As a result, a "ledge" is observed in the bias range of -0.3 to $-0.45 \mathrm{~V}$, and is more prominent for the lower frequency $C-V$ curves. Several other groups also observe a similar "ledge" in the $C-V$ curves for their metaloxide-semiconductor and metal-oxide-semiconductor field-effect transistor (MOSFET) structures. ${ }^{29-31}$ Using the capacitively detected magnetic resonance (CDMR) technique for their $p$-channel enhancement MOSFET device, Brandt et $a .^{29}$ concluded that the reason for the presence of such a ledge could be due to the defects caused by in-diffusion or

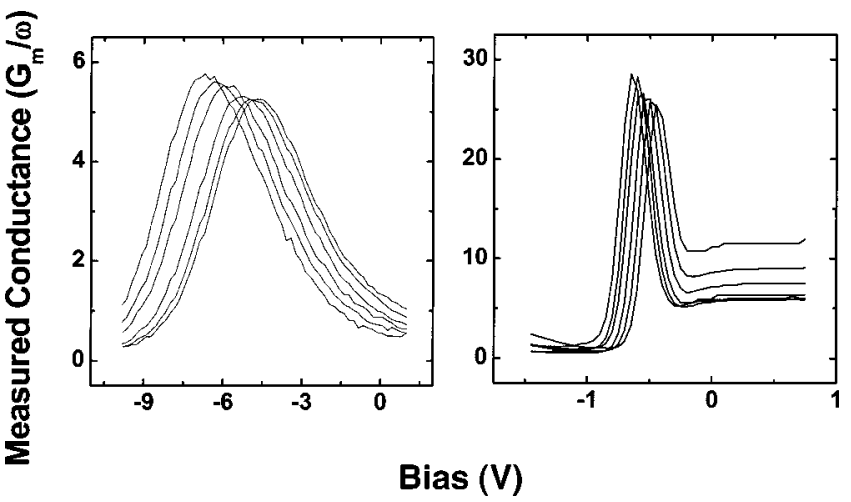

FIG. 5. Frequency dependence of the $G-V$ curves for (a) sample A, (b) sample B and sample A annealed in hydrogen (inset). Frequencies shown are $10,20,40,100,200$, and $400 \mathrm{kHz}$ and $1 \mathrm{MHz}$ left to right.

implantation of transition metal impurities. Returning to the case in hand, as was mentioned in the optical analysis, the carbon impurities may be responsible for introducing defects, which respond to the applied frequency. However, we have observed a similar "ledge" in the $C-V$ curves for AlN films deposited by magnetron sputtering of an $\mathrm{Al}$ target in $\mathrm{N}_{2} /$ Ar plasma. These films do not have any carbon contamination. We believe that there is nitrogen incorporation in silicon with subsequent bonding of $\mathrm{Si}-\mathrm{N}$ resulting in an interlayer formation. The defects created by nitrogen incorporation in silicon may be responsible for the "ledge" in the $C-V$ curve.

The frequency dependence of the $C-V$ curves for sample B annealed in hydrogen gas, shown in Fig. 4(b), revealed considerable reduction in the ledge that appeared in the as-deposited sample at lower frequencies probably due to the passivation of defects created by nitrogen incorporation in silicon. An overall reduction of frequency dispersion in the $C-V$ curves compared to that shown in Fig. 4(a) was also observed. Interestingly the frequency dispersion in accumulation has reduced to $\sim 4.5 \%$. This is probably due to restoration of dielectric loss as a result of annealing. ${ }^{32}$ Presence of free radicals in a dielectric is believed to be one of the reasons responsible for dielectric losses. Thermal annealing results in a decrease in the free radical concentration preventing reaction with $\mathrm{O}_{2}$ and the formation of polar groups. The $G-V$ curves were once again sharply peaked as were the ones obtained for the as-deposited sample indicating that the losses are predominantly due to interface states.

Figures 5(a) and 5(b) show the conductance-voltage $(C-V)$ characteristics for samples A and $\mathrm{B}$, respectively, corresponding to the respective $C-V$ curves in Figs. 3 and 4(a). The peaks of the $G-V$ curves are shifted according to the flatband voltage shift of the $C-V$ curves. The frequency dispersion in the accumulation region of $G-V$ curves of sample B corresponds to the dispersion in the accumulation region of relevance in the $C-V$ curves, which has been explained before. While the $G-V$ curves of sample A are stretched corresponding to the $C-V$ curves, the $G-V$ curves of sample B are sharply peaked. It is interesting to note that the magnitude of the loss peaks in Fig. 5(a) changes by only $\sim 7 \%$ and that in Fig. 5(b) changes by only $\sim 9 \%$ over the entire frequency range, indicating that the time constant dispersion is not very large. In fact, detailed analysis indicated a 


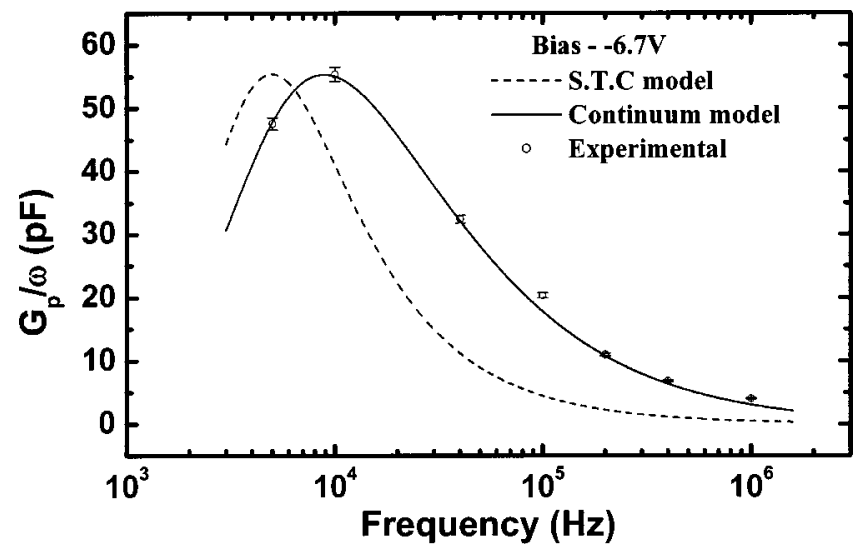

FIG. 6. Equivalent parallel conductance $G_{p} / \omega$ vs frequency for sample A.

total absence of anomalous time constant dispersion

Figures 6 and 7 are the plots of normalized equivalent parallel conductance versus frequency for samples A and B, respectively, at the specified biases. The curve fitting was done as follows: $G_{p} / \omega$ was extracted from the measured capacitance and conductance using the method explained by Nicollian and Goetzberger. ${ }^{7,8}$ At each fixed bias, the maximum value of $G_{p} / \omega$, when plotted against frequency, went through a maximum. This value and the corresponding frequency were recorded. These values are then substituted in Eq. (1) to evaluate $D_{\text {it }}$ based on the single time constant model and into Eq. (2) to evaluate $D_{\text {it }}$ based on a continuum model. ${ }^{33}$ These values are resubstituted into Eqs. (1) and (2), respectively, to generate theoretical $G_{p} / \omega$ versus frequency plots. The experimental points were then fitted into the plots. Figure 6 shows that over the range of bias investigated, $G_{p} / \omega$ versus frequency showed good fit to theory if a continuum of states is assumed. We observed the same type of behavior at all values of gate bias in the depletion region. Figure 7 shows that best fitting for sample B was obtained if the single time constant model is assumed. In this study the single time constant behavior is observed at gate

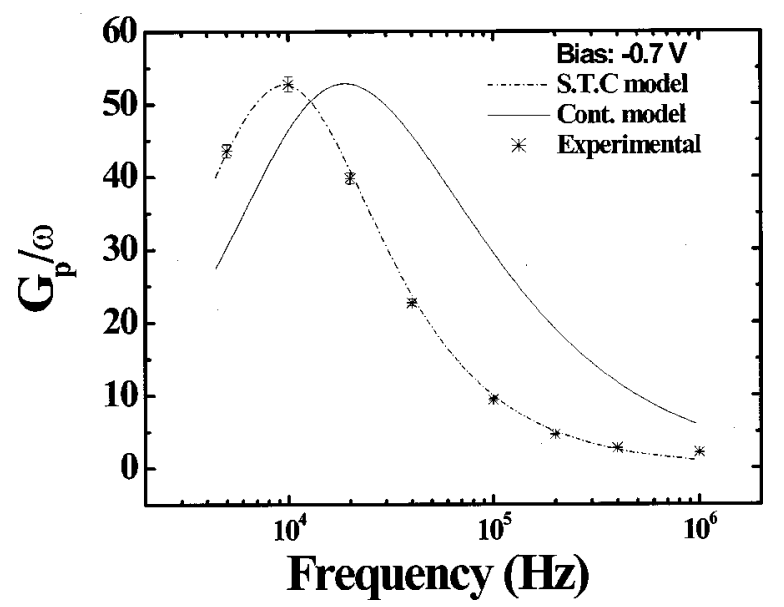

FIG. 7. Equivalent parallel conductance $G_{p} / \omega$ vs frequency for sample B.

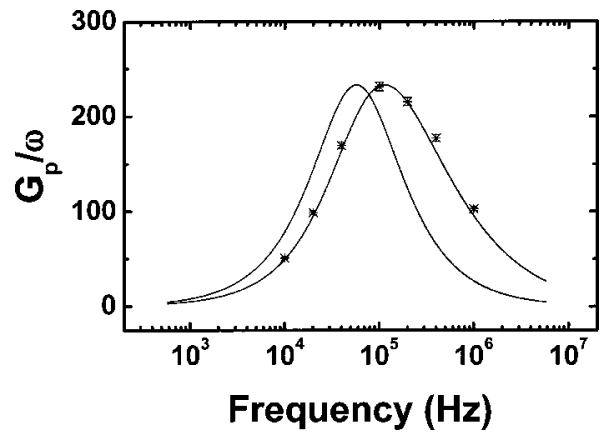

FIG. 8. Equivalent parallel conductance $G_{p} / \omega$ vs frequency for the ledge region of sample $\mathrm{B}$.

bias range of -0.75 to $-0.50 \mathrm{~V}$. Interestingly the plot of $G_{p} / \omega$ versus frequency in the bias range -0.35 to $-0.45 \mathrm{~V}$ (the ledge region) fits the theoretical curve if only the continuum model is assumed. Figure 8 shows such a plot at $-0.35 \mathrm{~V}$.

Figure 9 shows a plot of interface state density $\left(D_{\text {it }}\right)$ versus energy for samples A, B, and sample B annealed in hydrogen. $D_{\text {it }}$ for Sample A was calculated to be $\sim 4.23$ $\times 10^{11} \mathrm{~cm}^{-2} \mathrm{eV}^{-1}$ at the midgap. Continuum of interface trap levels, as observed in the case of sample A, could result from chemical inhomogeneity at the Si/insulator interface. ${ }^{18}$ The inhomogeneity is caused due to stretched, bent, or broken bonds between the insulator and the Si surface. In our case such a chemical disorder in the vicinity of the Si surface may be caused due to chemical bonding of $\mathrm{Si}-\mathrm{N}$ resulting in a stretched or broken bond which cause spreading of interface traps in the Si band gap.

For sample B, at the midgap the interface state density was calculated to be $1.59 \times 10^{11} \mathrm{eV}^{-1} \mathrm{~cm}^{-2}$. The $D_{\text {it }}$ versus energy plot for this sample is characterized by the presence of a "hump" in the bias range of -0.30 to $-0.45 \mathrm{~V}$. This alters the usual semihyperbolic nature ${ }^{18}$ of the curve usually obtained for $D_{\text {it }}$ versus energy plots. Interestingly, the observed hump corresponds to the deep-depletion regime (steep slope) of the observed ledge in Fig. 4(a). The fact that $D_{\text {it }}$ peaks at a certain bias $(-0.45 \mathrm{~V})$ value is indicative of an increase in dangling bonds at the insulator-silicon interface about $0.2 \mathrm{eV}$ away from the conduction band.

Although considerable work has been published regarding the processing of AlN films, not much literature is avail-

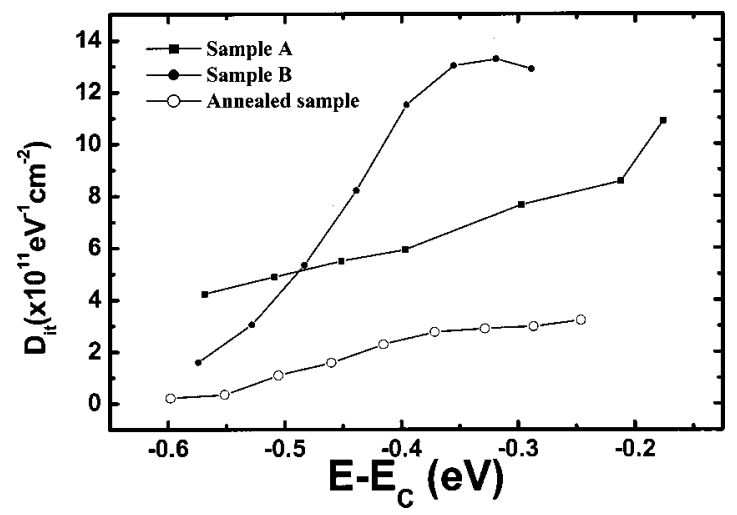

FIG. 9. Interface state density vs energy for samples A, B, and A annealed in hydrogen. 


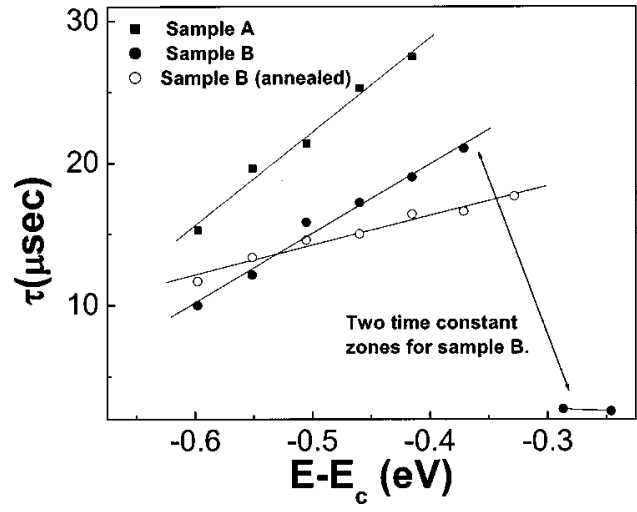

FIG. 10. Time constants vs bias for samples A, B, and A annealed in hydrogen.

able with the analysis of the substrate/insulator interface. For comparison therefore we present the $D_{\text {it }}$ values of some other insulators with similar dielectric constants. Chave et al. reported a $D_{\text {it }}$ of $\sim 1 \times 10^{11} \mathrm{~cm}^{-2} \mathrm{eV}^{-1}$ for their $\mathrm{Al} / \mathrm{Al}_{2} \mathrm{O}_{3} / \mathrm{InP}$ MISFET structure. ${ }^{34}$ Basa et al. reported a $D_{\text {it }}$ of $\sim 1.96$ $\times 10^{12} \mathrm{~cm}^{-2} \mathrm{eV}^{-1}$ for their $\mathrm{Al} / \mathrm{SiN}_{x} / \mathrm{Si}$ MIS configuration. ${ }^{35}$

The $D_{\text {it }}$ at midgap for the sample annealed in hydrogen was calculated to be $3.37 \times 10^{10} \mathrm{~cm}^{-2} \mathrm{eV}^{-1}$. This is almost an order of magnitude less than that obtained for the asdeposited sample D. A hump is observed again but it is not as sharply peaked as in the case of as-deposited samples. A comparison of $D_{\text {it }}$ versus energy plot for sample B and the hydrogen annealed sample indicates that not only the $D_{\text {it }}$ value at midgap is lower for the annealed sample, but also that the increase in the $D_{\text {it }}$ values as one goes towards the conduction band is slow. This is similar to that observed in MOS devices annealed in hydrogen. As stated earlier, interface traps arise from unsatisfied dangling bonds at the surface of the semiconductor due to abrupt termination of the silicon surface along a given plane. Logically, the formation of the insulator ties up some but not all of the Si-surface bonds. When the sample is annealed in hydrogen, the hydrogen species migrates through the insulator layer to the insulator-Si interface where it attaches itself to a dangling $\mathrm{Si}$ bond thereby making the bond electrically inactive. This causes a reduction in the interface state density for the sample annealed in hydrogen.

The time constants of the interface traps were evaluated using conditions on $\omega \tau$ stated before. Figure 10 is the plot of time constants of the interface states versus bias for samples A, B, and sample B annealed in hydrogen. For sample A no abnormal dispersion of time constants was observed. This corresponds well with the $G_{p} / \omega$ plot, which peaked in a close spectrum around $10 \mathrm{kHz}$ for all the biases corresponding to the depletion region. This is surprising in view of the fact that the $\mathrm{SiO}_{2}-\mathrm{Si}$ interface exhibits a large time constant dispersion in the depletion region. Of interest is the fact that some of the points are scattered around the straight line. This is due to the fact that the HP4275A measures conductance only at a few discrete values of frequency. Hence the exact value at which the conductance peaks for a given value of gate bias cannot be located with certainty.

The plot of time constants of the interface states versus

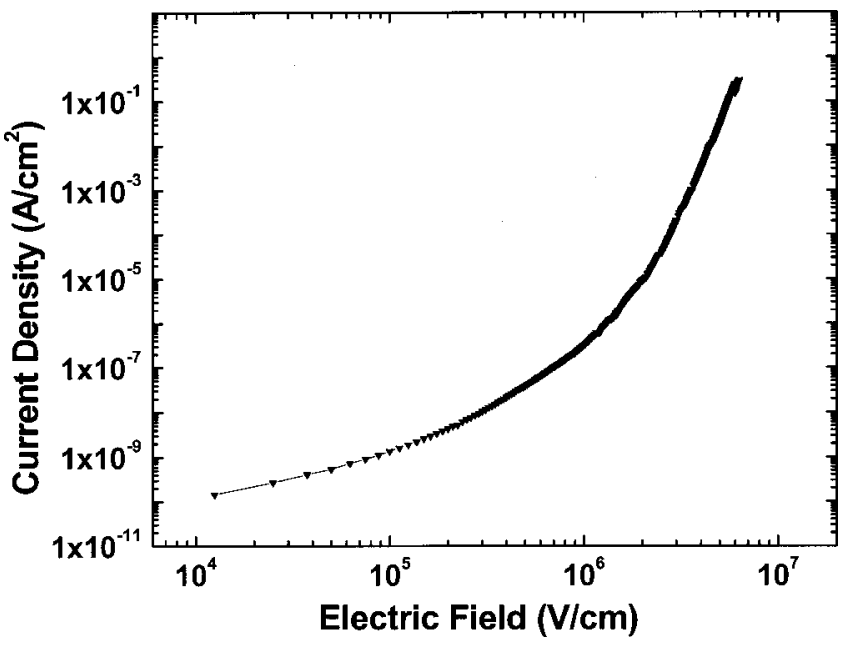

FIG. 11. Current density vs electric field for sample B.

bias for sample $\mathrm{B}$ in the bias range of -0.35 to $-0.80 \mathrm{~V}$ indicates two distinct regions of time constants: (1) in the bias range of -0.35 to $-0.45 \mathrm{~V}$ pertaining to the ledge region of the $C-V$ curve and (2) in the bias range of -0.45 to $-0.80 \mathrm{~V}$. Interestingly, annealing in hydrogen results in the usual straight-line plot of time constants versus bias, as shown in Fig. 10.

The defects caused by probable nitrogen incorporation influences the time constants of the interface traps about 0.2 $\mathrm{eV}$ away from the conduction band resulting in faster traps. Furthermore, the fact that $G_{p} / \omega$ values fit the continuum model in this bias range indicates that a region at the insulator-silicon interface has become chemically inhomogenous.

Breakdown voltage, leakage current, and complete current-voltage $(I-V)$ characteristics were measured on all the samples. Figure 11 shows the current density versus applied bias for a typical AlN sample. To obtain a current transport mechanism, $\ln (J / E)$ versus $\sqrt{ } E$ was plotted. This showed a linear relationship at high fields. This implies that current conduction at high fields is a bulk-limited process and is dominated by a Frenkel-Poole conduction. ${ }^{6}$ From the linear portion of the plot, a dielectric constant of $9.2 \pm 0.3$ was calculated. This is well within the predicted range of $8-10$ for AlN films.

\section{SUMMARY AND CONCLUSION}

Deposition of AlN films that are amorphous in nature has been completed using a metalorganic precursor in a conventional PECVD configuration. The nature of the film varies from carbon-free hydrogenated AlN, which displays optical properties that are similar to those of AlN from other methods, to carbon-rich films with AIN incorporated into its structure. The primary controlling factors for the quality of the AlN films is the rf power and the process pressure. Subsequent annealing of the carbon-rich films correlates the evolution of $\mathrm{H}$ from the film with the change in the carbon structure.

The overall electrical characteristics strongly depended on the processing conditions. The AlN samples showed good 
bulk and interfacial properties. The total ac loss is small and a first order calculation yields $D_{\text {it }}$ values that are reasonable from a device point of view. It is especially interesting to note that the AlN/silicon interface does not exhibit a large dispersion of interface state time constants commonly associated with a $\mathrm{SiO}_{2} / \mathrm{Si}$ interface. Annealing in hydrogen showed marked improvement in the interfacial properties and the value of the interface state density was observed to reduce by almost an order of magnitude. From our study of PECVD deposited aluminum nitride films it can be concluded that with optimization of processing parameters it is possible to make device grade AlN films.

${ }^{1}$ S. A. Nikishin et al., Appl. Phys. Lett. 75, 484 (1999).

${ }^{2}$ F. C. Steadile, I. J. R. Baumvol, W. H. Schriener, and F. L. Friere, J. Vac. Sci. Technol. A 10, 3272 (1992).

${ }^{3}$ W. J. Meng, J. A. Sell, T. A. Perry, L. E. Rehn, and P. M. Baldo, J. Appl. Phys. 75, 3446 (1994).

${ }^{4}$ H. Okano, N. Tanaka, Y. Takahashi, T. Tanaka, K. Shibata, and S. Nakano, Appl. Phys. Lett. 64, 166 (1994).

${ }^{5}$ K. Yasutake, A. Takeuchi, H. Kakiuchi, and K. Yoshii, J. Vac. Sci. Technol. A 16, 2140 (1998).

${ }^{6}$ S. M Sze, Physics of Semiconductor Devices, 2nd ed. (Wiley, New York, 1981).

${ }^{7}$ E. H Nicollian and J. R Brews, MOS Physics and Technology (Wiley, New York, 1982).

${ }^{8}$ E. H Nicollian and A Goetzberger, Bell Syst. Tech. J. 46, 1055 (1967).

${ }^{9}$ F. Hasegawa et al., Jpn. J. Appl. Phys., Part 1 26, 1555 (1987).

${ }^{10} \mathrm{~S}$. Bhat et al., J. Electron. Mater. 14, 405 (1985).

${ }^{11}$ A. Ishizaka and Y. Shiraki, J. Electrochem. Soc. 133, 666 (1986).

${ }^{12}$ J. Bauer, L. Biste, and D. Bolze, Phys. Status Solidi A 39, 173 (1977).

${ }^{13}$ A. Boudrioua, D. Remiens, A. Boudrioua, and J. Loulergue, Appl. Phys. Lett. 74, 1209 (1999).

${ }^{14}$ H. Nomura, S. Meikle, Y. Nakanishi, and Y. Hatanaka, J. Appl. Phys. 69, 990 (1991).
${ }^{15}$ J. Loretz, B. Despax, P. Marti, and A. Mazel, Thin Solid Films 265, 15 (1995).

${ }^{16}$ L. Interrante, W. Lee, M. McConnell, N. Lewis, and E. Hall, J. Electrochem. Soc. 136, 472 (1989).

${ }^{17}$ R. Krupitskaya and G. Auner, J. Appl. Phys. 84, 2861 (1998).

${ }^{18}$ D. R. Kerr, J. S. Logan, P. J. Burkhardt, and W. A. Pilskin, IBM J. Res. Dev. 8, 376 (1964).

${ }^{19}$ E. H. Snow and M. E. Dumensil, J. Appl. Phys. 37, 2123 (1966).

${ }^{20}$ E. H. Snow and B. E. Deal, J. Electrochem. Soc. 113, 263 (1966).

${ }^{21}$ B. E. Deal, P. J. Flemming, and P. L. Castro, J. Electrochem. Soc. 115, 300 (1968).

${ }^{22}$ S. Logothetidis, E. Evangelou, and N. Konofaos, J. Appl. Phys. 82, 5017 (1997).

${ }^{23}$ H. Hasegawa, K. E. Forward, and H. Hartnagel, Appl. Phys. Lett. 26, 567 (1975).

${ }^{24}$ A. Azim Khan, J. A. Woollam, and Y. Chung, Solid-State Electron. 27, 385 (1984).

${ }^{25}$ M. Mizuta, S. Fujieda, T. Jitsukawa, and Y. Matsumoto, Inst. Phys. 83, 153 (1986).

${ }^{26}$ A. H. Khan, J. M. Messe, T. Stacy, E. M. Charlson, E. J. Charlson, and G. Zhao, Mater. Res. Soc. Symp. Proc. 339, (1994).

${ }^{27}$ T. Mandel, M. Frischolz, R. Helbig, S. Birkle, and A. Hammerschimdt, Appl. Surf. Sci. 65/66, 795 (1993).

${ }^{28}$ R. F. Pierret, Semiconductor Device Fundamentals (Addison-Wesley, New York, 1996), p. 597.

${ }^{29}$ M. S. Brandt, R. T. Neuberger, and M. Stutzmann, Appl. Phys. Lett. 76, 1467 (2000)

${ }^{30}$ H. Fu and C. T Sah, IEEE Trans. Electron Devices ED-19, 15 (1972).

${ }^{31}$ K. M. Brunson, D. Sands, C. B. Thomas, and H. S. Reehal, J. Appl. Phys. 60, 3599 (1986).

${ }^{32}$ H. Yasuda, Plasma Polymerization (Academic, New York, 1985).

${ }^{33}$ A. Azim Khan, J. A. Woollam, Y. Chung, and B. Banks, IEEE Electron Device Lett. 6EDL-4, (1983).

${ }^{34}$ J. Chave, A. Choujaa, C. Santinelli, R. Blanchet, and P. Viktorovitch, J. Appl. Phys. 61, 257 (1987).

${ }^{35}$ D. K. Basa, M. Bose, and D. N. Bose, J. Appl. Phys. 87, 4324 (2000). 